\author{
Revista de Psicología Vol. 34 (1), 2016 (ISSN 0254-9247) \\ http://dx.doi.org/10.18800/psico.201601.004
}

\title{
Itinerarios, hitos y catalizadores asociados a la emergencia del talento docente ${ }^{1}$
}

\author{
María Caridad García-Cepero², Félix Antonio Gómez-Hernández³, Darcy \\ Milena Barrios-Martínez ${ }^{4}$, Andrea Santamaría ${ }^{5}$, Laura Estefanía Castro \\ Fajardo $^{6}$, Andrea Sánchez Vallejo ${ }^{7}$ y Zulma Patricia Zuluaga Ocampo ${ }^{8}$ \\ Pontificia Universidad Javeriana, Colombia
}

Este artículo presenta los resultados de la fase de exploración y contextualización del proyecto "Rutas de emergencia del talento docente" estudio de caso en maestros con un uso sobresaliente de las TIC. Este es un estudio mixto-secuencial explicativo. En la primera fase se clasifica a los participantes utilizando un análisis de correspondencia múltiple; y, en la segunda y fase principal, se analizan tres entrevistas diferentes realizadas a nueve maestros, utilizando el modelo de Gagné (2009, 2015) como marco analítico para el análisis de contenido de ellas. Esta fase identifica itinerarios, figuras y eventos significantes en las historias

1 Se describen algunos de los resultados del Proyecto "Rutas de emergencia del talento docente", financiado por la Pontificia Universidad Javeriana, la Fundación Compartir y la Fundación Telefónica.

2 Doctora en Psicología Educativa por la Universidad de Connecticut y profesora asociada a la Facultad de Educación de la Pontificia Universidad Javeriana. Dirección postal: Facultad de Educación, Pontificia Universidad Javeriana Cra. 7 No. 40-62 Edif. 25, Bogotá. Contacto: maria.caridad@javeriana.edu.co

3 Magíster en Educación y profesor asistente en la Pontificia Universidad Javeriana de Bogotá. Contacto: felixantoniogh@gmail.com

4 Magíster en Educación y profesora de cátedra en la Facultad de Educación de la Pontificia Universidad Javeriana de Bogotá. Dirección postal: Calle 51 sur \# 36-71, Bogotá, Colombia. Contacto: milebama17@yahoo.com

5 Licenciada en Pedagogía Infantil por la Pontificia Universidad Javeriana. Contacto: a-santamaria@javeriana.edu.co

6 Estudiante de Licenciatura en Pedagogía Infantil y Licenciatura en Lenguas Modernas de la Pontificia Universidad Javeriana. Contacto: lcastrof@javeriana.edu.co

7 Magíster en Educación y profesora de cátedra en la Facultad de Educación de la Pontificia Universidad Javeriana de Bogotá. Contacto: asanchezv@javeriana.edu.co.

8 Magíster en Lingüística Española del Instituto Caro y Cuervo y profesora asistente de la Facultad de Educación de la Pontificia Universidad Javeriana. Contacto: zzuluaga@javeriana.edu.co 
de los participantes, los cuales son determinantes para comprender la emergencia del talento docente.

Palabras clave: talento docente, educación, calidad educativa, prácticas excepcionales, estudio de caso, TIC.

Itineraries, milestones and catalysts associated with the emergence of talent of professors This document presents the results of the exploration and contextualization phase of the project "Rutas de emergencia del talento docente, estudio de casos en maestros con un uso sobresaliente de las TIC" ("Teaching talent emergency paths, case study in teachers with outstanding use of ICTs"). It is a mixed sequential explicative study. The first phase classifies participants utilizing a multiple correspondence analysis. The second phase performs and analyzes three different interviews to nine teachers, utilizing the Gagné model (2009, 2015) to analyze content. This phase identifies itineraries, figures, and significant events in the participants' histories, which can understand the emergence of teaching talent in the Colombian context.

Keywords: gifted teachers, education, educational quality, exceptional teaching, case study, ICT.

\section{Percursos, etapas e catalisadores associados com o surgimento do talento docente}

Este artigo apresenta os resultados da fase de exploração e contextualização do projeto "rotas de emergência do talento docente: estudos de caso com professores com uso excelente das TIC”. Esta é uma pesquisa mista-sequencial explicativa. Na primeira fase os participantes foram classificados usando um analise múltiplo de correspondências; e na segunda fase, foram analisadas três entrevistas diferentes realizadas a nove docentes, usando como marco para o analise de conteúdo das mesmas, o modelo de Gagné (2009, 2015). Esta fase identifica roteiros, figuras e eventos significativos em as histórias dos entrevistados, as quais são determinantes para compreender a emergência do talento docente.

Palavras-chave: talento docente, educação, qualidade educativa, práticas excepcionais, estudo de caso, TIC. 
A pesar de los crecientes estudios que buscan caracterizar los perfiles de maestros y maestras con altos desempeńos, como se observa por ejemplo en los trabajos citados por Harris (2010) de Stevenson y Stigler, (1999), Darling-Hammond (2000), Bain, (2006), Barber y Mourshed (2008), Kukla-Acevedo (2009), Kane, Rockoff, y Staiger (2006), Aaronson, Barrow y Sander (2007), así como aquellos de Harris y Sass (2011), Pérez, Casas, Vargas e Isaza (2014), todavía es poco lo que se sabe en torno a los procesos y los factores asociados que hacen factible la emergencia de sus producciones excepcionales. No parece excesivo afirmar, entonces, que hasta el momento ha sido más fácil identificar el talento docente que explicar el porqué de su emergencia. Esto podría deberse a que la sociedad actual exige de forma continua innovaciones, pero se preocupa poco por develar los factores que hacen posible aquello mismo que demanda.

Es evidente, por el contrario, en panoramas como el de la ciencia o el arte, que las realizaciones sobresalientes no son el resultado de procesos aleatorios o el producto de mecanismos que escapen a la explicación científica, sino que tienen su origen tanto en la forma en que ciertas personas capitalizan sus capacidades como en las circunstancias que hacen posible que emerja el acto creador que innova, como se observa en los trabajos de Simonton (1997, 1999a, 1999b, 2013), así como en los estudios de Gardner (2011) y Kaufman (2013). De igual manera, se sabe que estas capacidades se refinan con la experiencia y el conocimiento y, en consecuencia, no son privativas de unos pocos, sino que pertenecen al acervo cognitivo que comparten todos los seres humanos (Renzulli, 1978, 2012; Renzulli \& Delcourt, 2012).

En tal sentido, es de gran importancia avanzar en una interpretación en torno a las rutas recorridas por los maestros y maestras, con el objetivo de comprender cómo se origina y desarrolla el talento docente, puesto que es en las historias de vida de las personas donde se entretejen 
las capacidades intelectivas, los intereses, las influencias sociales y culturales, las capacidades creativas y el conocimiento adquirido (Callahan \& Hertberg-Davis, 2012; Gagné, 2005, 2012, 2015). Desde esta perspectiva, el talento docente debe comprenderse como una propiedad emergente y no estructural de la acción docente que se traduce en la construcción de ideas o acciones educativas innovadoras.

Considerado así el asunto, este estudio se concentró en un campo promisorio de investigación: el referido a los procesos de enseñanzaaprendizaje mediados por la tecnología. Este énfasis se debe a que hoy, naturalmente, se espera que el conjunto total de profesores puedan incorporar las TIC como una herramienta de mediación en los procesos educativos, así como integrar a sus estudiantes en esta dinámica.

Por ello, esta primera aproximación investigativa sobre talento docente estará centrada en maestros que se han destacado por hacer un uso pedagógico sobresaliente de las tecnologías. Al hablar de sobresaliente se utiliza como criterio el hecho de que la experiencia pedagógica presentada por el docente fue valorada por un panel de expertos como destacada al compararla con las experiencias presentadas por sus pares, lo que condujo a su nominación al Premio Compartir y al Premio Telefónica, o a la selección entre todos los docentes participantes en las Aulas Telefónica.

Si bien se tiene claro que el proceso de enseñanza-aprendizaje mediado por la tecnología no es el único campo de en el que el talento docente se puede manifestar, se advierte que este es un escenario relevante para construir acciones educativas innovadoras, y resulta pertinente de cara a los desafíos de la Sociedad de la Información.

\section{La excelencia docente en el marco del desarrollo del talento}

Una forma de aproximarse al problema de la excelencia docente es comprenderlo desde el marco del desarrollo del talento y las capacidades excepcionales (gifted and talent development). Tannembaum (1983) plantea que es difícil saber con precisión por qué algunos desempeños 
extraordinarios son considerados excepcionales y otros no; no obstante, es claro para el autor que las sociedades valoran ciertos comportamientos excepcionales y activamente buscan su emergencia. Por su parte, Sternberg, Jarvin \& Grigorenko (2010) consideran que se estaría en presencia de un talento cuando se puede establecer que quien lo manifiesta presenta un desempeño superior en alguna dimensión o conjunto de dimensiones en relación con sus pares, cumpliendo con cinco criterios: excelencia, rareza, productividad, demostrabilidad y valor.

\section{Desarrollo del talento y la excepcionalidad}

Desde el origen de los estudios sobre capacidades y talentos excepcionales a finales del siglo XIX, han surgido múltiples concepciones sobre a qué llamar talento y su relación con las capacidades excepcionales o la superdotación. Los estudios iniciales se ligaron más al problema de la genialidad y, poco a poco, los autores fueron utilizando acepciones distintas como talento y excepcionalidad (aunque en algunos casos los utilizaron indistintamente).

$\mathrm{Al}$ analizar:

Las diferentes concepciones sobre talento y sus conceptos relacionados como superdotación, dotación, 'giftedness', 'high-ability', entre otros, es posible concluir que no existe en la actualidad un acuerdo sobre estos términos y que, dependiendo del autor, cultura y momento histórico, se han definido de manera diferenciada o indistinta" (Sternberg \& Davidson, 1986; Bralic \& Romagnoli, 2000; García-Cepero \& González, 2004; Mayer, 2006; Kaufman \& Sternberg, 2008; citados en García-Cepero \& Proestakis, 2010, p. 37).

De allí que algunas de las concepciones contemporáneas y liberales sobre el talento estén asociadas a atributos cognitivos y a atributos no cognitivos que facilitan la expresión y el desarrollo del potencial de los sujetos, como se observa en la Figura 1. 


\section{Elementos involucrados en concepciones contemporáneas del talento académico / dotación}

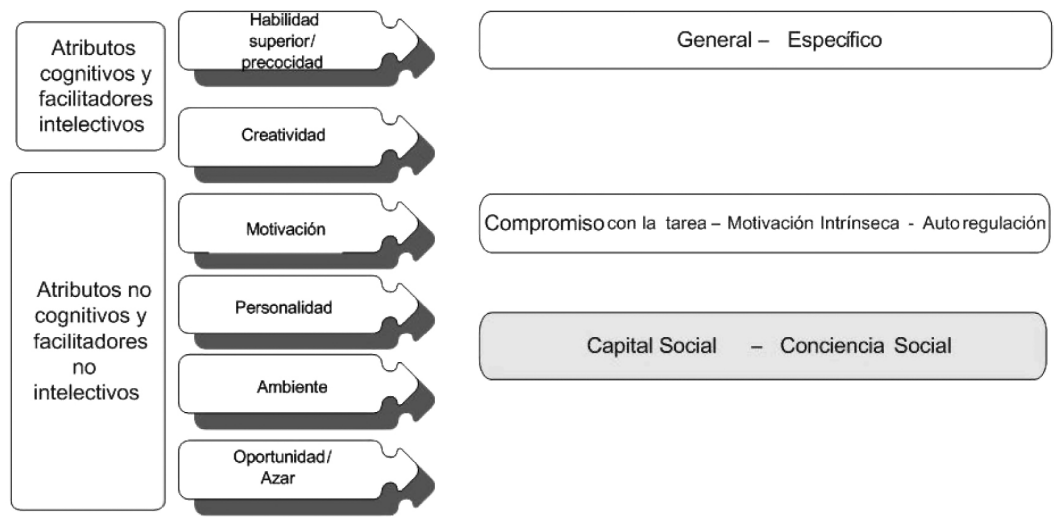

Figura 1. Elementos involucrados en las concepciones de talento (García-Cepero \& Proestakis, 2010, p. 39)

Por ende, puede afirmarse que no hay un criterio absoluto o correcto, sino un continuo de comprensiones que van desde posturas esencialistas del talento, hasta posturas desarrollistas del mismo (Dai, 2010; Dai \& Chen, 2014). En ese sentido, esta investigación construye una aproximación a la concepción de talento docente desde una perspectiva desarrollista, y particularmente emergentista, pues se considera que para que el talento se manifieste, no solo se requiere de que el potencial se desarrolle, sino también que existan unas condiciones en el medio que permitan que este pueda evidenciarse en producción o ejecución, creativas o expertas.

\section{Una nueva aproximación al concepto de talento}

El hecho de que durante más de un siglo este campo se haya enfrentado a diferentes tensiones explica que la discusión actual radique en el análisis de las diversas aproximaciones al problema del desarrollo del talento. En esta línea, una primera tensión es el paso del concepto de 
superdotación al concepto de talento, por cuanto el CI como indicador de talento es una medida muy limitada para comprender la producción creativa excepcional. En segundo lugar, se encuentra la tensión entre el talento y la salud mental, pues del estudio del talento como un problema clínico de adaptación del individuo con altas capacidades, se transitó al análisis de las condiciones ambientales acordes a las necesidades de la persona para poder desarrollar su potencial. La tercera tensión surge debido a que algunos autores proponen que el talento/ excepcionalidad no es un atributo estructural de la persona, sino de su comportamiento; dicho de otro modo, no se debe hablar del "talento", sino de "emergencia o manifestación del talento" en el comportamiento de un individuo (Renzulli, 1978, 1980, citado en García-Cepero \& González, 2004).

Una cuarta tensión indica que la emergencia del talento es coyuntural, es decir, que se presenta en algunas personas, bajo ciertas circunstancias y en ciertos momentos de su vida (Renzulli, 1978, 1980, citado en García-Cepero \& González, 2004). Por otra parte, existe una quinta tensión que separa el concepto de "capacidades excepcionales" del de "talento", proponiendo que el término capacidades excepcionales/dotación/giftedness designa la posesión y uso de capacidades naturales destacadas, en tanto que el de talento hace referencia al dominio sobresaliente de capacidades sistemáticamente desarrolladas, llamadas competencias (conocimientos y destrezas), que sólo se desarrollan bajo el efecto de diversos aspectos ambientales. Estos aspectos se denominan "catalizadores" y se categorizan en tres grupos: por un lado, los catalizadores intrapersonales (atributos físicos, motivación volición, autogestión, personalidad); por otro, los catalizadores ambientales (medios, personas, recursos, sucesos) y, finalmente, el azar que, en su conjunto, permiten la transformación de las habilidades naturales en habilidades sistemáticamente desarrolladas que se cristalizarán en diferentes campos específicos como se observa en la Figura 2 (Gagné, 2005). 


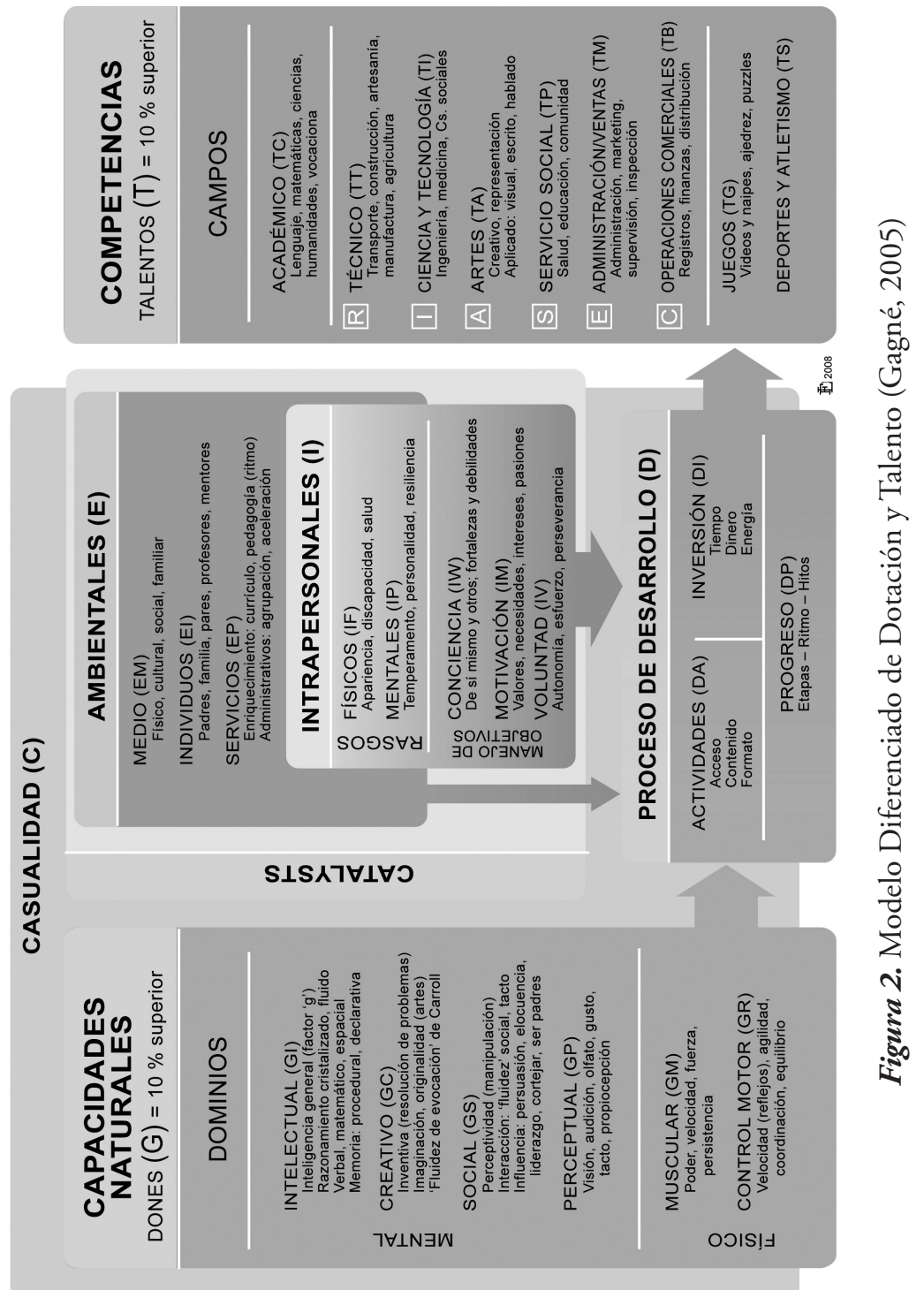


Para finalizar, un último punto de tensión se centra en el sentido del desarrollo del talento; esta situación puede ser resuelta en el marco de las propuestas del modelo de capacidades de Amartya Sen y Martha Nussbaum, desde donde se define "[...] el rendimiento y el éxito en función de las oportunidades que se abren a cada persona" (Nussbaum, 2011, p. 33).

En el contexto de las seis tensiones descritas anteriormente se desarrolla este proyecto, donde el talento se entenderá como: (a) la manifestación de un dominio destacado de capacidades sistemáticamente desarrolladas en términos de producción creativa o ejecución experta en un campo específico; (b) como una propiedad emergente de la acción del sujeto, que es coyuntural y que se manifiesta en algunas personas, bajo ciertas circunstancias y en ciertos momentos de su vida; y (c) como un dominio determinado por factores cognitivos, no cognitivos, personales, sociales y estocásticos, cuyo sentido está en el desarrollo de condiciones por parte de la sociedad, para que el individuo pueda tener una vida prolongada, saludable y creativa, que a la vez contribuya al desarrollo de la comunidad.

\section{La emergencia del talento docente y la construcción de capacidades}

Explorar el talento docente supone indagar por la responsabilidad que tiene la sociedad en la generación de las condiciones que permitan que este emerja. Siguiendo esta línea, el Estado y los sistemas educativos, desde la formulación de políticas públicas hasta su implementación, evaluación y perfeccionamiento en el seno de las comunidades, son quienes tienen el encargo principal de garantizar que los ciudadanos posean las herramientas, escenarios y medios para el desarrollo del talento, pues desde perspectivas actuales debe concebirse a "cada persona como un fin en sí mismo" y además, debe velarse "[...] por las oportunidades disponibles para cada ser humano" (Nussbaum, 2012, p. 38). En consonancia con esto, Nussbaum (2012) plantea la noción de "capacidades" no únicamente referida a las habilidades, aptitudes o destrezas con las que cuentan los seres humanos, 
sino que alude también a las combinaciones que surgen entre estas y el ambiente en el que se encuentran inmersos (espacios académicos, sociales y políticos).

En términos de la presente investigación, el talento docente se considera como una propiedad emergente en las personas, por lo cual se estaría hablando del talento como el resultado de una serie de capacidades combinadas que se manifiestan en la construcción de ideas o acciones educativas innovadoras, especialmente en el uso pedagógico ejemplar de las tecnologías al interior de las prácticas educativas.

Sin embargo, como ya se ha expuesto anteriormente, para que el talento emerja deben existir unas condiciones específicas que permitan a las personas elegir libremente lo que quieran hacer y ser en determinados momentos de su vida. En consecuencia, la sociedad debe crear circunstancias y ambientes en los que no se transgreda la calidad de vida de los sujetos; sino que, por el contrario, alimente sus esfuerzos y deseos, dentro de una ética del cuidado del intelecto, la persona y la sociedad.

Por ende, el presente artículo tiene como propósito primordial presentar los resultados del estudio "Rutas de emergencia del Talento Docente" relacionados específicamente con el estudio de los factores que están asociados a la emergencia del talento de los docentes que hacen un uso pedagógico sobresaliente de las tecnologías. Con todo lo antedicho, esta investigación busca ser pionera en la comprensión de las realizaciones excepcionales de los docentes, puesto que hasta el momento muy pocos autores han comprendido que la "excelencia docente" es una clara evidencia de la manifestación del talento docente.

\section{Método}

En el marco de la investigación: Rutas de emergencia del Talento Docente, se utilizó un diseño explicativo secuencial - DEXPLIS, que consiste en una primera fase de recolección y análisis de datos cuantitativos, seguida de una segunda fase de datos cualitativos 
(construida a partir de la primera fase). Esto implicó un diseño de naturaleza mixta, en el que se integraron técnicas de recolección y análisis de información tanto cualitativa como cuantitativa. El núcleo central de la investigación fue de estudio de caso y las fases iniciales permitieron recolectar la información para seleccionar de la muestra inicial los mejores candidatos que mostrando la diversidad de antecedentes, demostraran muy altos niveles de excelencia docente.

\section{Participantes}

Para esta investigación se contó con una muestra inicial de 65 docentes, 56 de los cuales fueron invitados al estudio por haberse destacado en el Premio Compartir al Maestro en el área de Tecnología e Informática y otras áreas, incorporando tecnología, o por haber sido nominados al Premio Telefónica. Los nueve participantes restantes fueron nominados por Fundación Telefónica por las experiencias que desarrollan en Aulas Telefónica en sus instituciones de origen.

El premio Compartir al maestro es un reconocimiento que otorga la Fundación Compartir a docentes, quienes con apoyo de sus rectores se nominan pues considera que tienen prácticas excepcionales sobresalientes. Entre los docentes participantes se galardonan aquellas experiencias que muestran condiciones de excelencia y pueden ser consideradas ejemplares a nivel nacional. De estos 56 docentes 42 respondieron a la invitación para contestar la encuesta sobre los antecedentes académicos y profesionales de los maestros. En la tabla 1 es posible observar las características generales de los participantes en el estudio.

\section{Medidas e instrumentos de medición}

Para recolectar la información se realizaron cinco entrevistas semiestructuradas individuales, acompañadas con actividades basadas en técnicas de cartografía social (Herrera \& Flores, 2007; Paulston \& Liebman, 1995). La información presentada en este artículo se basa en tres entrevistas realizadas a cada participante y en la ejecución de un grupo focal. 


\section{Tabla 1}

Características de las muestras utilizadas en el estudio

\begin{tabular}{|c|c|c|c|}
\hline Fase & $\begin{array}{c}\text { Fase de } \\
\text { contextualización }\end{array}$ & $\begin{array}{l}\text { Fase de } \\
\text { exploración }\end{array}$ & $\begin{array}{c}\text { Fase de } \\
\text { profundización }\end{array}$ \\
\hline $\begin{array}{l}\text { Fuente de } \\
\text { información }\end{array}$ & $\begin{array}{l}\text { Experiencias - } \\
\text { Bases de datos }\end{array}$ & Encuestas & Entrevistas \\
\hline $\begin{array}{l}\text { Tamańo de la } \\
\text { muestra }\end{array}$ & $\begin{array}{l}65 \text { docentes - } \\
64 \text { experiencias }\end{array}$ & 42 docentes & 9 docentes \\
\hline $\begin{array}{l}\text { Origen de los } \\
\text { participantes }\end{array}$ & $\begin{array}{l}\text { 86\% Premio } \\
\text { Compartir - } \\
\text { 14\% Aulas } \\
\text { Telefónica }\end{array}$ & $\begin{array}{l}79 \% \text { Premio } \\
\text { Compartir - } \\
21 \% \text { Aulas } \\
\text { Telefónica }\end{array}$ & $\begin{array}{l}78 \% \text { Premio } \\
\text { Compartir - } \\
22 \% \text { Aulas } \\
\text { Telefónica }\end{array}$ \\
\hline $\begin{array}{l}\text { Género de los } \\
\text { participantes }\end{array}$ & $\begin{array}{l}56 \% \text { hombres - } \\
44 \% \text { mujeres }\end{array}$ & $\begin{array}{l}57 \% \text { hombres - } \\
43 \% \text { mujeres }\end{array}$ & $\begin{array}{l}56 \% \text { hombres - } \\
44 \% \text { mujeres }\end{array}$ \\
\hline $\begin{array}{l}\text { Área de desempeño } \\
\text { del participante }\end{array}$ & $\begin{array}{l}32 \% \text { área } \\
\text { tecnología - } \\
68 \% \text { otras áreas }\end{array}$ & $\begin{array}{l}36 \% \text { área } \\
\text { tecnología - } \\
64 \% \text { otras áreas }\end{array}$ & $\begin{array}{l}11 \% \text { área } \\
\text { tecnología - } \\
89 \% \text { otras áreas }\end{array}$ \\
\hline $\begin{array}{l}\text { Sector de la } \\
\text { institución } \\
\text { donde trabaja el } \\
\text { participante }\end{array}$ & $\begin{array}{l}73 \% \text { oficial - } \\
27 \% \text { privado }\end{array}$ & $\begin{array}{l}72 \% \text { oficial - } \\
28 \% \text { privado }\end{array}$ & $\begin{array}{l}67 \% \text { oficial - } \\
33 \% \text { privado }\end{array}$ \\
\hline $\begin{array}{l}\text { Zona de la } \\
\text { institución del } \\
\text { participante }\end{array}$ & $\begin{array}{l}16 \% \text { rural - } \\
84 \% \text { urbano }\end{array}$ & $\begin{array}{l}10 \% \text { rural - } \\
90 \% \text { urbano }\end{array}$ & $100 \%$ urbano \\
\hline $\begin{array}{l}\text { Región de la } \\
\text { institución del } \\
\text { participante }\end{array}$ & $\begin{array}{l}40 \% \text { Bogotá - } \\
60 \% \text { otras } \\
\text { ciudades }\end{array}$ & $\begin{array}{l}42 \% \text { Bogotá } \\
-58 \% \text { otras } \\
\text { ciudades }\end{array}$ & $\begin{array}{l}33 \% \text { Bogotá - } \\
67 \% \text { otras } \\
\text { ciudades }\end{array}$ \\
\hline
\end{tabular}


Las entrevistas de historia de vida tuvieron como propósito recolectar datos biográficos de los entrevistados desde los que se pudieran determinar factores asociados a la emergencia del talento docente: (a) la experiencia, que indagó por la forma en que surgió la experiencia, su desarrollo, los factores que han permitido su cualificación y la manera como se llevó a cabo la incorporación de las tecnologías a la experiencia; y (b) la cartografía, segmento en que se buscó conocer la trayectoria docente seguida por el entrevistado e identificar los principales hitos, conocimientos/competencias y catalizadores determinantes en su carrera como docente.

\section{Procedimiento y procesamiento de información}

Con base en los datos recogidos sobre antecedentes de los maestros (formación y motivos para ingresar a la carrera docente), se identificaron cinco tipos de rutas de entrada a la carrera docente, esto a través de un análisis de correspondencias múltiples.

En la fase de profundización se seleccionaron nueve casos que ilustraban las rutas identificadas; el propósito de esta selección era que la muestra total diera cuenta de las diferentes entradas a la carrera docente. Así, los nueve maestros seleccionados para el estudio de caso participaron en cinco entrevistas semiestructuradas centradas en los elementos fundamentales de la investigación (historia de vida, cartografía de la carrera docente, descripción de la experiencia nominada, presentación de un portafolio sobre los elementos representativos de su práctica, uso y concepción de tecnología), y un grupo focal.

La información derivada de esta fase de recolección de datos se transcribió y analizó con la ayuda del software Nvivo 10. Para este proceso, se realizó un análisis de contenido mixto con base en categorías deductivo-inductivas centrado en las categorías centrales del modelo de Gagné (2005) ilustradas en la Figura 2. Durante el proceso de codificación, se permitió la incorporación de categorías inductivas que aparecían como temas recurrentes en los casos (el autoaprendizaje, los saberes, el papel de los estudiantes, entre otras categorías). 


\section{Resultados}

\section{Origen, itinerarios e hitos en la emergencia del talento docente}

$\mathrm{Al}$ realizar el análisis de las líneas de tiempo que plasmaban las nueve trayectorias de los docentes se encontró que presentan grandes diferencias, lo cual, en parte, es de esperar pues los nueve casos fueron seleccionados para que representaran maestros que ingresan a la carrera docente por motivaciones diversas. Por ejemplo, tres de los participantes fueron conscientes de su interés en la carrera docente a edades muy tempranas, cuatro de ellos en su adolescencia y dos en su juventud.

Dice mi mami que desde que yo era muy pequeña acostumbraba a jugar con mis amigos a que yo era la profesora. Entonces, ¿qué era lo que pasaba?, invitaba a mis amigos, obviamente todos: "Ya nos va a decir que es la profesora”, aburridísimos todos, yo cogía de tablero la pared y entonces empezaba: "Yo soy la profesora, que yo les voy a dictar una clase, que yo les voy a enseñar, niños pónganme atención" (P3, Entrevista Cartografía).

No soy como la mayoría, que desde pequeño quiso ser docente, yo encontré mi vocación tiempo después porque alguien me dijo que tenía madera para ser docente, desde ahí visioné la docencia para ser un proyecto de vida y empecé a trabajar realmente sobre ese proyecto, me di cuenta que ser docente ya era lo mío (P), Entrevista Cartografía).

Algunos los perciben como un llamado (vocación) pero otros lo ven en el marco de la profesionalización; sin embargo, en la gran mayoría de casos, se integran ambas visiones.

Pero para mí, ser maestro la principal diferencia es la vocación, es decir, yo me siento maestra no porque para mí sea algo de qué vanagloriarme sino porque como que ha estado en mí siempre, yo amo esto, esto es mi mundo y yo lo hago como lo disfruto un montón yo soy feliz [...] (P7, Entrevista Cartografía).

[...] de alguna manera había como un cierto interés por parte mío, pero también una presión social, de dignificar el tema de la docen- 
cia; como que no era cualquier actividad, como que yo también estaba estudiando una carrera profesional. Y entonces, como dar el valor a lo pedagógico en ese sentido (P2, Entrevista Cartografía).

Por otra parte, algunos entrevistados inician su educación formal como docentes en escuelas normales, otros en programas de licenciatura y otros vienen desde campos profesionales diferentes. En adición, algunos tienen su primera experiencia como docentes antes de iniciar el pregrado y otros de manera posterior a este; pero en todos, sin excepción, la primera experiencia de aula es definitiva y se constituye en uno de los principales hitos que, a criterio de ellos, determina quiénes son como docentes:

Cuando uno se gradúa de la Universidad tú traes una cantidad de información y de teorías y de metodologías así incluso escritas y trabajas por gente que nunca ha estado con los niños, pero cuando te vas al campo y entras en contacto con ellos y los empezáis a conocer y ves la realidad, todas esas teorías te dan unos fundamentos teóricos, pero la verdad te haces maestro es allí viviendo, haciendo, adquiriendo esa experiencia del contacto, del conocer, del intentar, de tener que sentarte a llorar con los nińos en muchas ocasiones o de sus alegrías y sus cosas (P9, Entrevista Cartografía).

En contraste con lo anterior, si bien todos los docentes hacen alusión en sus entrevistas a su paso por la universidad (pregrado y postgrado), en los discursos no es evidente que este paso resulte significativo más allá de la adquisición de conocimientos y el encuentro con algunos docentes que sí impactan en su desarrollo profesional. Este factor nos lleva a cuestionar si dichos espacios están en alguna medida subutilizados en tanto catalizadores del proceso de emergencia del talento docente. También, es de señalar que, a excepción de aquellos que tuvieron formación como normalistas, el espacio de practicum aparece ausente como evento significativo.

Un segundo hito que se presenta reiterativamente en todos los entrevistados es la participación en redes académicas, ya sea en grupos de investigación (casos 7 y 8), redes temáticas (casos 4 y 9), equipos 
interdisciplinarios (casos 2, 3, 5 y 7) y equipos de área (casos 1, 5, 7 y 9). Esta participación en redes se constituye en una estructura de acogida para generar capacidades combinadas de emergencia de talento, pues en las redes se aprende, crea, debate y reflexiona, sobre el hacer y el ser de la docencia:

[...] era otra forma de trabajo que me llamó mucho la atención y le mueve el piso a uno como docente, y al moverle el piso como docente uno tiene que empezar a cambiar paradigmas, a reflexionar y a darse cuenta con humildad que otros le pueden enseñar y eso, pues, lo logramos con varios docentes que estaban en la red (P9, Entrevista Cartografía).

Un último hito que se presenta recurrentemente, en buena medida por la naturaleza de la muestra del estudio, es el de los reconocimientos. Todos los participantes han recibido de alguna manera reconocimientos externos a su institución por las experiencias excepcionales que están realizando. El reconocimiento juega un papel determinante en el proceso de identificación de talento docente; pero, por otra parte, en los participantes se observó un impacto de catalización positiva de este factor.

Y a partir de este proceso hemos tenido la posibilidad de mostrarle a la ciudad lo que hacemos; se genera una propuesta desde la Alcaldía de Bogotá para atender a los niños en condición de enfermedad; empiezo a liderar un proyecto piloto; empiezo a vincular a estudiantes en un tema de práctica social, proyectos de tesis de diferentes universidades que realmente lo que hacen es contribuir y mejorar el servicio que nosotros ofrecemos para esos niños en condición de enfermedad (P2, Entrevista Cartografía).

Este impacto se representa, por una parte, en la validación y retroalimentación sobre su propia práctica; por otra, en un espacio de producción creativa donde el docente socializa las innovaciones pedagógicas que desarrolla $y$, finalmente, en alguna medida, como una oportunidad de adquirir recursos que le permiten dar continuidad a las experiencias. En pocas palabras, se constituye en un motor para seguir produciendo prácticas excepcionales. Es así que espacios como 
el Premio Compartir al Maestro, el Premio Telefónica, entre otros, son estructuras de acogida, que al igual que el primer trabajo docente y las redes de académicas permiten generar capacidades combinadas para la emergencia del talento docente.

El Premio Compartir me marcó, claro, me hizo tomar conciencia de que lo que yo hacía era no bueno, sino significativo. A partir de ahí cada semestre cambio... Entonces eso me llevó a recrear, a cambiar constantemente la práctica didáctica y pedagógica para formar con sentido social (P4, Entrevista Cartografía).

Con Fundación Telefónica he podido acceder a plataformas virtuales, las capacitaciones que nos dan me generan más ideas... es un aliado a un sueño. El sueño que tenía mi institución, que se refleja en unas políticas, en una filosofía, en una misión y visión, en una postura que tenía el director del Hospital Pediátrico conectó con lo que me resuena a mí en la vida (P2, Entrevista Cartografía).

A esto se añade que en las entrevistas fue posible identificar aproximadamente 114 referencias a personas que fueron determinantes en la carrera docente, siendo en su orden: familia y pares académicos con $21 \%$ de las referencias cada uno, sus docentes (17\%), directivos y estudiantes (15\% cada uno) y otros agentes (11\%).

El papel de los pares está claramente vinculado a la generación de redes como estructuras de acogida, como fue descrito previamente y se complementa con la siguiente afirmación: "Al descubrir en ellos digamos unas facultades que yo no poseo, como unas inteligencias más desarrolladas y más alcances, digamos otras visiones del mundo porque ciertas inteligencias le permiten a uno ver más allá” (P5, Entrevista Cartografía).

El papel que juega la familia está enraizado en aspectos que determinan catalizadores intrapersonales como la personalidad, la motivación y los valores que rigen el quehacer de los docentes. Se identifican dos atributos reiterativos en las estructuras familiares: altas expectativas de logro y apoyo. En adición, se encuentra una valoración positiva del trabajo docente y del proyecto de vida de sus hijos. 
Algo muy lindo, por ejemplo de mi mamá, es la apuesta que ella le hizo a mis sueños. Nunca me dijo no, no haga eso o no estudie eso. Hace poco me decía ella que ella no entendía muy bien por qué yo había elegido el tema de la educación especial, porque ella también tenía la idea de que eran niños ya saben cómo, pero que ella respetaba mucho, y siento que gracias a ese silencio oportuno que tuvo yo no entré en un conflicto mucho más complejo del que estuve (P2, Entrevista Cartografía).

En tanto a los directivos, en alguna medida se constituyen como facilitadores e interlocutores en la construcción de propuestas que son la puesta en escena donde emerge el talento o en figuras formadoras y ejemplo a seguir:

La directora es una persona que tiene una vocación increíble para llegarle al estudiante, para guiarlo, para hacerlo crecer y para ayudar a sus padres a formarlo [...] Entonces esta persona, me llegó su discurso y su esencia y su persona como tal, me llegó hartísimo. Entonces elegí esta opción (la opción educativa) (P6, Entrevista Cartografía).

En algunos casos son enunciados como figuras negativas, pero esto no se constituye en impedimento para la emergencia del talento, siempre y cuando la persona cuente con atributos personales (perseverancia, autoconfianza, etc.) que compensen la falta de apoyo.

Por otra parte, es importante resaltar los múltiples roles que tienen los estudiantes en el proceso de emergencia del talento docente. Un primer punto es que los estudiantes se constituyen en motor y motivo para la generación de innovación en su práctica pedagógica, pues en todos los casos estudiados, la innovación surge a partir de problemáticas de los estudiantes, bien sean de aprendizaje o de su contexto social. En palabras de uno de los docentes: "Sí, porque es que sin los niños yo no sería nada, sería un proyecto vacío" (P8, Entrevista Cartografía).

Un segundo aspecto bajo esta mirada, es que los estudiantes se convierten en pares en el proceso de aprendizaje y, en alguna medida, en maestros. Esto se hace particularmente evidente en lo relacionado con el uso de la tecnología: 
Con los muchachos porque uno solo no. A veces los muchachos le ganan a uno, entonces por ejemplo: que vamos a hacer un sitio web y allá nadie sabía en el pueblo, entonces uno aprendía y los muchachos también aprendían; nos colocábamos a hacer tutoriales y nos enseñábamos (P1, Entrevista Cartografía). Los niños son esponjas que absorben y mientras más les das más absorben y entonces fue maravilloso ahí estuve siete años y ahí aprendí muchísimo, muchísimo (P7, Entrevista Cartografía).

\section{Catalizadores ambientales, catalizadores intrapersonales y azar: una relación sincrónica}

En el desarrollo del análisis de contenido fue posible identificar en todas las historias de vida la mayoría de catalizadores planteados por Gagné (2015), lo cual hablaría de la pertinencia de este modelo como marco analítico para comprender la emergencia del talento docente. La Figura 3, resume los principales hallazgos en cuanto a la identificación de catalizadores.

Tanto los eventos significativos descritos previamente como las personas significativas se constituyen en algunos de los elementos propios de los catalizadores ambientales. En adición a estos, se hacen manifiestos los espacios de aprendizaje que, en términos de Gagné (2015), hacen referencia a los servicios educativos. Dentro del proceso de codificación no se hizo manifiesto alguno de estos, puesto que deberían corresponder a procesos generados intencionalmente por las instituciones y el sistema educativo para crear capacidades de emergencia del talento. En este sentido, es importante cuestionarnos qué tipo de programas se podrían implementar para favorecer la emergencia del talento docente.

En cuanto a los atributos que describen a aquellos docentes significativos durante el proceso formativo de los maestros entrevistados se identifican atributos como la apertura (generación de espacios para ejercer la creatividad), acompañado de altos niveles de exigencia, pasión, trabajo en equipo, preocupación más allá de lo programático y testimonio, se encontraron discursos como el siguiente: 

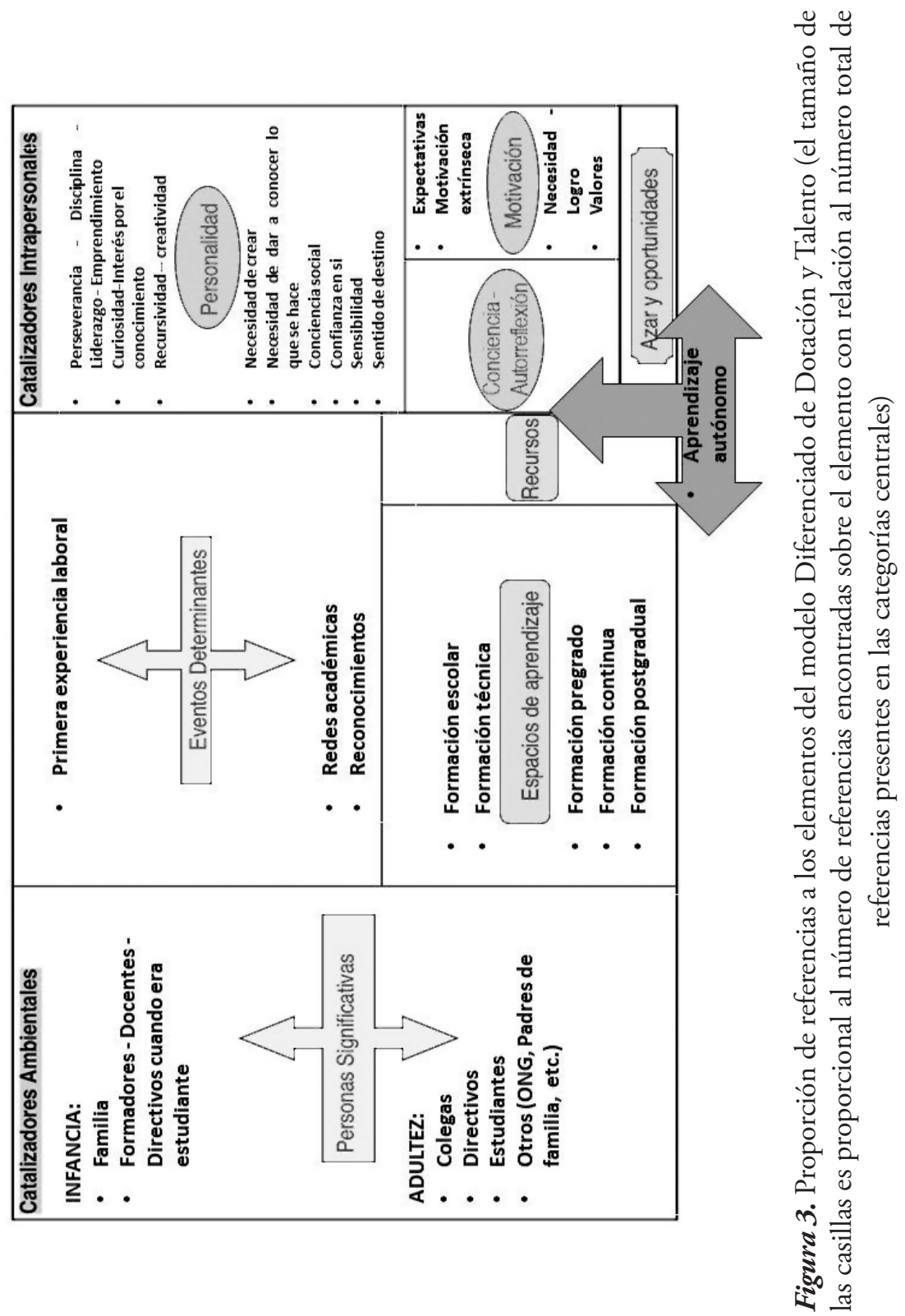
Es que tenía mucha admiración por ella, era profesora de biología y química. Era de las personas más creativas, uno no se aburría en clase, siempre llevaba actividades diferentes, era con la profesora con la que podíamos salir de la institución, íbamos a excursiones, se sentaba con uno "ven te explico, es que esto es asî", tenía sus libros y decía "pues llévatelos, léetelos"; entonces eso fue como que muy chévere y creo que muchos del grupo (que éramos un grupo pequeño como de veinte personas) de los que estábamos de directora de grupo en octavo optamos por seguir porque ella nos apasionó y era como que muy rico y hablaba de la pedagogía, la didáctica, nos capturó para que no nos fuéramos a las otras instituciones (P8, Entrevista Cartografía).

Un elemento adicional que se puede observar en este punto es que al menos en la mitad de los participantes hay evidencia de que experiencias positivas del ámbito educativo los orientaron a iniciar la carrera educativa y, en varios de ellos, precisamente fue uno de sus docentes quien le sugirió y animó a perseguir este proyecto de vida. Esto permite señalar que los docentes de educación básica, media y superior, no sólo son ejemplo, sino también son, de manera voluntaria o involuntaria, partícipes en procesos de identificación de talento tácitos o explícitos y, por esta razón, debe potenciarse su labor.

$\mathrm{Al}$ analizar las referencias hechas al contexto laboral fue posible identificar temas recurrentes en cuanto a la gestión directiva, como son: la apertura y apoyo para el desarrollo de proyectos innovadores (o que salgan de lo estipulado programáticamente), el favorecimiento del trabajo en equipo y desarrollo de proyectos, una cultura de exigencia y rendimiento de cuentas, así como procesos de gestión claros y eficaces y, finalmente, liderazgo con ejemplo. Todo ello se corresponde con varios de los atributos encontrados en investigaciones que caracterizan buenas prácticas de gestión directiva (McCoach et al., 2010).

Un elemento fundamental en la emergencia del talento docente son los catalizadores intrapersonales. En el estudio, se hacen evidentes algunos rasgos típicos de los participantes, que se manifiestan en casi todos ellos, como son la perseverancia, la disciplina, el liderazgo, el emprendimiento, la recursividad, la creatividad, la necesidad de crear y 
de dar a conocer lo creado, así como un marcado interés por el conocimiento. Estos elementos concuerdan altamente con los atributos planteados por diferentes autores que tienden a estar asociados con el desarrollo del talento en otros campos Gagné (2015).

No fue posible observar en las entrevistas las capacidades naturales que subyacen a la emergencia del talento docente, posiblemente porque la naturaleza de la técnica de recolección utilizada no lo favorece; sin embargo, se pudo observar la presencia de altas habilidades sociales, así como creativas e intelectuales, que corresponden a lo planteado por Gagné (2015). En contraste, los participantes frecuentemente mostraron la importancia de saberes como el pedagógico-didáctico, las competencias investigativas, las competencias en uso de tecnología y el conocimiento de los estudiantes y su contexto. Estos aspectos son consistentes con las características descritas por Porath (2009) que caracterizan la experticia que requiere un docente talentoso.

Este interés por el conocimiento, unido con la experticia, posiblemente esté altamente asociado con un catalizador que surge inductivamente y es el autoaprendizaje. Todos los docentes participantes en los estudios de caso muestran como tema recurrente la presencia de aprendizajes autónomos. Estos están claramente ligados a la construcción de propuestas de innovación y a la necesidad de responder a las problemáticas de los estudiantes como se señaló previamente.

Cuando te tienes que documentar por ejemplo, para preparar un curso, yo he volteado muchísimos cursos, cuando yo llegué a la Universidad el curso me lo daban: "bueno este es el curso" entonces yo me puse a leer eso, eran documentos, entréguele a los estudiantes trescientas fotocopias de... y "bueno, ¿cómo vas en la clase?" y yo llegaba les entregaba, "siéntense, lean", el profesor se sentaba acá un rato y bueno, "¡listo, tiempo! y vamos a discutirlo" y eso terrible [...] no, yo dije: “¿qué es eso?” yo me moriría de aburrimiento y entonces: "bueno listo, sí claro, necesito los teóricos, necesito los libros, necesito los documentos [...]” (P7, Entrevista Cartografía).

La presencia de un proceso de aprendizaje autorregulado recurrente puede ser un claro indicador de la presencia de talento docente, 
pues en muchas de las definiciones de talento, por ejemplo la desarrollada por Ellen Winner (s/f, citada en MEN, 2006), se incluye entre los atributos propios de los talentos específicos la automaestría en los campos específicos.

En cuanto a los aspectos de motivación y volición se hacen evidentes unas claras expectativas en los docentes, ligadas a la necesidad de logro y a las motivaciones intrínsecas. Sin embargo, en varios de los docentes se hace evidente cómo motivaciones extrínsecas son interiorizadas al punto que se vuelven propias.

Finalmente, un catalizador intrapersonal recurrente en todos los participantes es la conciencia y la autorreflexión, en particular, asociadas con sus prácticas de aula y el mejoramiento de las mismas. Esto se puede ilustrar en lo que para el siguiente participante significó la experiencia de ser entrevistado para este estudio:

Hoy que me están invitando a hacer una reconstrucción de mi historia, pero probablemente en cada uno de estos caminos he tenido crisis y entonces también me dejo llevar por mis miedos, pero siento que también he tenido la posibilidad de revisarlos. No solamente a lo formativo académicamente, sino las experiencias que hoy me invitan a tener una intención clara desde mi proyecto de vida de que quiero ser docente. Ha sido uno de los elementos más representativos; ha sido integrarlo con mi proyecto de vida personal y que tenga un significado y que me sienta feliz de hacer lo que hago (P2, Entrevista Cartografía).

Para finalizar este análisis es importante explorar el papel del azar en el proceso de emergencia del talento docente. Uno de los cambios que ha tenido el modelo de Gagné (2015) desde sus inicios es el desplazamiento del factor de azar o casualidad en la emergencia del talento docente. Según el autor (Gagné, 2015), inicialmente el azar o casualidad era considerado como un factor catalizador en el desarrollo del talento; sin embargo, poco a poco se ha convertido en un factor que en buena medida se encuentra mediado por la agencia personal y los catalizadores intrapersonales. Es así como en varios de los casos se puede pensar que, en parte, el talento emerge porque el docente está en el 
lugar apropiado y en el momento apropiado. Sin embargo, es importante entender el azar como una oportunidad que el docente tiende a aprovechar en función de la construcción de innovaciones pedagógicas.

Ejemplo de esto es la experiencia del siguiente participante, quien aprovecha las características propias del municipio para, a partir de ellas, generar procesos educativos innovadores y trascendentes:

Después se nos ocurrió lo de la memoria histórica y ese sí dio excelentes resultados, pero entonces, ¿qué hacíamos de la memoria histórica?, nos propusimos rescatar la memoria histórica del pueblo, que había sido unos de los tres lazaretos que existían en Colombia para albergar a los enfermos de lepra. Yo decía bueno, esa historia no la tiene cualquiera, la tenemos tres en este país y nadie más (P1, Entrevista Cartografía).

\section{Discusión}

Del análisis presentado es importante resaltar tres puntos importantes. El primero, es la pertinencia tanto del concepto de talento docente como el de algunos modelos (desarrollados por autores como Gagné) para comprender los factores asociados, que hacen viable la emergencia de producciones excepcionales en el campo educativo. Es claro que muchos de los aspectos que los autores del campo de las capacidades y talentos excepcionales señalan son aplicables para el talento docente.

Las conceptualizaciones realizadas alrededor del quehacer de los docentes han evolucionado y se han enriquecido a través de los años. Ejemplo de esto es lo planteado por autores como Larrosa (2010) o Tenti (2010), quienes muestran la evolución y las tensiones que surgen frente a la concepción de la docencia como oficio, como vocación y como profesión.

Dentro de este panorama, la presente investigación quiere plantear una nueva compresión de la docencia desde el marco de las concepciones de talento, en la medida que, comprender la excelencia en este 
campo y desde esta perspectiva, integra tanto los elementos volitivosmotivacionales y de mística, propios de las concepciones centradas en la docencia como vocación, con los elementos de experticia que están típicamente presentes en las aproximaciones centradas en la profesionalización. Adicionalmente, reconoce tanto la producción como la ejecución creativa y experta, a manera de manifestaciones del talento docente y, dadas las concepciones contemporáneas del talento, su comprensión es desarrollista y sistémica. Dicho en otras palabras, no centra el problema de la excelencia docente exclusivamente en el docente mismo y sus capacidades internas y básicas (entendidas desde la perspectiva de Nussbaum, 2012), sino que, también se focaliza en la construcción de capacidades combinadas.

Por otra parte, la concepción de la docencia como un campo en el cual el talento se puede manifestar, contribuye a la dignificación del quehacer docente, siendo coherente con los discursos actuales de la política educativa que ubican la excelencia docente como un factor determinante en la calidad educativa, que a su vez, es uno de los factores más potentes en el desarrollo de las naciones (García, Maldonado, Perry, Rodríguez \& Saavedra, 2014).

Como último punto de reflexión de este estudio es importante resaltar el "círculo virtuoso" que se desencadena cuando se crea la capacidad en las aulas para que los maestros manifiesten talento docente; los docentes sobresalientes toman el ejemplo de otros docentes sobresalientes, y a su vez pueden constituirse en ejemplo de vida para los estudiantes, como señala uno de los participantes en el estudio, quien resalta el papel de la educación como factor que contribuye a superar las condiciones de pobreza:

¿Qué te hace a ti un docente atípico? [...] yo creo que el grado de pobreza que yo viví en mi infancia me llevó a buscarle solución definitiva a esa pobreza y yo entendí por la visión y por eso admiro a mis tíos porque en ese momento eran los referentes que yo tenía y ellos eran profesores, y ellos eran buenos profesores y son buenos profesores (bueno, ya mi tía ya se pensionó el año pasado) [...] Pero yo entendí que la única manera de salir de la pobreza es educarse 
y yo creo que eso es lo que busco venderles a mis estudiantes, que tengan otras opciones, que valoren la educación y que amen la escuela, que valoren la escuela y que quieran estar en la escuela y que quieran aprender. Entonces, si yo no les vendo y si yo no amo lo que estoy haciendo, ellos no lo van a amar, apasionarme como me apasiono es una forma de, también, involucrarlos a ellos y llevarlos a que se apasionen por la educación. [...] Yo creo que esa es la salida [...] entonces, yo les digo a mis estudiantes cuando están reacios a las cosas "igual que tú yo también llegaba, a lo mejor un poquito malgeniado, porque a lo mejor no desayuné, porque a lo mejor no hubo comida, pero si estamos acá aprovechando el espacio, entonces, aprendamos porque estar aquí es la oportunidad que tienes [...] y a todos nos tocó difícil” (P6, Entrevista Cartografía).

Es importante señalar que no es posible, ni se pretende plantear una relación directa causal de los factores analizados (itinerarios, hitos y catalizadores) en la emergencia del talento docente, sino que ellos actúan de manera sinérgica con las habilidades y conocimientos, posibilitando la manifestación del talento, ya sea en términos de creación o de ejecución.

El talento [...] es una propiedad emergente del comportamiento de una persona, dada una combinación coyuntural de factores [catalizadores]. En esta medida, la responsabilidad del sistema educativo es incrementar la probabilidad de que el talento se manifieste de manera tal que ayude a los estudiantes a capitalizar su potencial y mejorar su calidad de vida (García-Cepero, 2009).

En otras palabras, es función de la sociedad y sus instituciones, y en este caso del sistema educativo, generar capacidades combinadas materializadas en estructuras de acogida, que se inician desde el seno familiar, continuando por la escuela y demás espacios de formación, así como en las instituciones educativas que se constituyen en el espacio laboral donde el talento docente encuentra su nicho de crecimiento. Por ende, es función de todos los actores, nombrados en esta sección, generar intencionalmente capacidades para que en el futuro más docentes estén en posibilidad de manifestar talento docente. 
Finalmente, es relevante precisar algunos alcances y limitaciones propias de este estudio. En primer lugar, es necesario indicar que dado que el constructo talento docente ha tenido poco desarrollo en la literatura académica, esta investigación debe considerarse exploratoria. Es por ello que una de las metas de la investigación es configurar este concepto, como herramienta analítica pertinente para comprender las experiencias pedagógicas sobresalientes.

En segundo lugar, dado que esta investigación está centrada principalmente en estudios de caso, es importante establecer límites a los alcances de esta en cuanto a la posibilidad de generalizar y transferir los resultados. No obstante, es importante resaltar la pertinencia que tienen los estudios de caso para profundizar y dar sentido a constructos poco explorados como el de talento docente.

Por último, es importante señalar que este estudio se centra en el autorreporte de las experiencias de los participantes, por tanto, se puede presentar un sesgo de fuente única; en este sentido, se propone comprender los resultados desde la experiencia de los sujetos participantes. Asimismo, se espera que futuros estudios permitan validar los desarrollos conceptuales realizados en esta investigación, a los que se sugiere abordar muestras más amplias y utilizar fuentes de información diversas que permitan la triangulación de los resultados.

\section{Referencias}

Aaronson, D., Barrow, L. \& Sander, W. (2007). Teachers and student achievement in the Chicago public high schools. Journal of Labor Economics, 25(1), 95-135. http://dx.doi.org/10.1086/508733

Bain, K. (2006). Lo que hacen los mejores profesores universitarios. Universitat de València.

Barber, M. \& Mourshed, M. (2008). Cómo hicieron los sistemas educativos con mejor desempeño del mundo para alcanzar sus objetivos. Santiago de Chile: PREAL 
Bralic, S. \& Romagnoli, C. (2000). Niños y jóvenes con talentos: una educación de calidad para todos. Santiago de Chile: Dolmen

Callahan, C. M. \& Hertberg-Davis, H. L. (Eds.). (2012). Fundamentals of Gifted Education: Considering Multiple Perspectives. New York: Routledge.

Dai, D. Y. (2010). The Nature and Nurture of Giftedness: A New Framework for Understanding Gifted Education. Education \& Psychology of the Gifted Series. New York: Teachers College Press.

Dai, D. Y. \& Chen, F. (2013). Paradigms of Gifted Education: A Guide for Theory-Based, Practice-Focused Research. Wako: Prufrock Press

Darling-Hammond, L. (2000). How teacher education matters. Journal of teacher education, 51(3), 166-173. http://dx.doi. org/10.1177/0022487100051003002

Gagné, F. (2005). From gifts to talents. Conceptions of giftedness, 2, 98-119. http://dx.doi.org/10.1017/CBO9780511610455.008

Gagné, F. (2012). From Gifted Inputs to Talented Outputs. Fundamentals of Gifted Education: Considering Multiple Perspectives. New York: Routledge.

Gagné, F. (2015). Delos genes al talento: la perspectiva DMGT7CMTD. Revista de Educación, 368, 12-39.

Garcia-Cepero, M. C. (2009). Hacia una pedagogía centrada en el talento, una alternativa de mejoramiento total de la escuela. 3er Seminario regional de Talento Antofagasta y Calama.

Garcia-Cepero, M. C \& Proestakis, M. N. (2010). Perspectivas de Atención a los Estudiantes con Talento Académico; Una visión Global. En M. C. Garcia-Cepero (Ed.), Talentos en el Bicentenario; Educación para el desarrollo de estudiantes sobresaliente (pp. 37-46). Antofagasta: Centro de Investigación y Desarrollo de Talento DeLTA-UCN, Universidad Católica del Norte.

García-Cepero, M. C. \& González J. (2004). Fundamentos de educación para la excepcionalidad. Bogotá: Javegraf - Secretaria de Educación de Cundinamarca.

García, S., Maldonado, D., Perry, G., Rodríguez, C. \& Saavedra, J. E. (2014). Tras la excelencia docente, Cómo mejorar la calidad de la 
educación para todos los colombianos. Bogotá: Fundación Compartir.

Gardner, H. (2011). Leading minds: An anatomy of leadership. New York: Basic Books.

Harris, D. N (2010). What makes for a good teacher and who can tell? Washington: CALDER, The Urban Institute.

Harris, D. N. \& Sass, T. R. (2011). Teacher training, teacher quality and student achievement. Journal of Public Economics, 95(7), 798-812. http://dx.doi.org/10.1016/j.jpubeco.2010.11.009

Herrera, J. D. \& Flores I. A. (2007). La cartografía social: una alternativa metodológica para el desarrollo de observatorios locales en educación y pedagogía. Memorias del VI congreso internacional de investigación en educación y pedagogía. Bogotá: IDEP.

Kane, T. J., Rockoff, J. E. \& Staiger, D. O. (2008). What does certification tell us about teacher effectiveness? Evidence from New York City. Economics of Education Review, 27(6), 615-631. http://dx.doi.org/10.1016/j.econedurev.2007.05.005

Kaufman, S. B. \& Sternberg, R. J. (2008). Conceptions of giftedness. En S. Pfeiffer (Ed.), Handbook of Giftedness in Children (pp. 71-91). Tallahassee, FL: Springer. http://dx.doi.org/10.1007/978-0387-74401-8_5

Kaufman, S. B. (2013). The Complexity of Greatness: Beyond Talent Or Practice. Oxford University Press. http://dx.doi.org/10.1093/ acprof:oso/9780199794003.001.0001

Kukla-Acevedo, S. (2009). Do teacher characteristics matter? New results on the effects of teacher preparation on student achievement. Economics of Education Review, 28(1), 49-57. http://dx.doi.org/10.1016/j.econedurev.2007.10.007

Larrosa, F. (2010). Vocación docente versus profesión docente en las organizaciones educativas. Revista Electrónica Interuniversitaria de Formación del Profesorado, 13(4), 43-52.

McCoach, D. B., Goldstein, J., Behuniak, P., Reis, S. M., Black, A. C., Sullivan, E. E. \& Rambo, K. (2010). Examining the unexpected: Outlier analyses of factors affecting student achievement. 
Journal of Advanced Academics, 21(3), 426-468. http://dx.doi. org/10.1177/1932202X1002100304

MEN (2006) Orientaciones para la atención educativa a estudiantes con capacidades o talentos excepcionales. Recuperado de http://186.113.12.12/discoext/collections/0032/0008/0269 0008.pdf

Nussbaum, M. C. (2011). Creating capabilities. Massachusetts: Harvard University Press. http://dx.doi.org/10.4159/ harvard.9780674061200

Nussbaum, M.C. (2012). Crear capacidades: propuestas para el desarrollo humano. Barcelona: Paidós.

Paulston R. G. \& Liebman M. (1995). The promise of critical social cartography. Inter American Review of Educational Development (OAS), 119.

Pérez, M., Casas, C. R., Vargas, Á. P. \& Isaza, L. A. (2014). ¿Qué caracteriza a un docente destacado?: rasgos de la práctica en los primeros grados de la escolaridad. Revista Colombiana de Educación, 67, 171-199.

Porath, M. (2009). What makes a gifted educator? A design for development. International handbook on giftedness. Springer Netherlands, 825-837. http://dx.doi.org/10.1007/978-1-40206162-2_40

Renzulli, J. S. (1978). What makes giftedness? Phi Delta Kappan, 60(3), 180-184.

Renzulli, J. S. (2012). Reexamining the Role of Gifted Education and Talent Development for the 21st Century A Four-Part Theoretical Approach. Gifted Child Quarterly, 56(3), 150-159. http://dx.doi.org/10.1177/0016986212444901

Renzulli, J. S. \& Delcourt, M. A. (2012). Gifted behaviors versus gifted individuals. Fundamentals of Gifted Education: Considering Multiple Perspectives. 
Simonton, D. K. (1997). Creative productivity: A predictive and explanatory model of career trajectories and landmarks. Psychological Review, 104(1), 66. http://dx.doi.org/10.1037/ 0033-295X.104.1.66

Simonton, D. K. (1999a). Origins of genius: Darwinian perspectives on creativity. Oxford University Press.

Simonton, D. K. (1999b). Talent and its development: An emergenic and epigenetic model. Psychological review, 106(3), 435.

Simonton, D. K. (2013). Creative performance, expertise acquisition, individual differences, and developmental antecedents: An integrative research agenda. Intelligence, 45, 66-73.

Sternberg, R. J. \& Davidson, J. E. (Eds.). (1986). Conceptions of giftedness. Cambridge, MA: Cambridge University Press. http:// dx.doi.org/10.1017/cbo9780511778049

Sternberg, R. J., Jarvin, L. \& Grigorenko, E. L. (2010). Explorations in giftedness. Cambridge University Press.

Stevenson, H. W. \& Stigler, J. W. (1999). ¿Por qué los escolares de Asia oriental tienen alto rendimiento académico? Estudios Públicos, 76, 297-357. Recuperado de http://ww2.educarchile.cl/Userfiles/P0001\%5CFile\%5Cstevenson\%20Escolares\%20de\%20 Asia\%20y\%20rendimiento.pdf

Tannembaum, A. J. (1983). Gifted children: Psychological and educational perspectives. New York: Macmillan.

Tenti E. (Ed.). (2010). El oficio de docente: vocación, trabajo y profesión en el siglo XXI. Buenos Aires: Siglo XXI.

Recibido el 01 de junio de 2015 Aceptado el 27 de septiembre de 2015 